\title{
Mejoramiento del gel de surimi de anchoveta (Engraulis ringens) mediante adición de surimi de brazo de calamar gigante (Dosidicus gigas)
}

\author{
Improvement of anchovy surimi gel (Engraulis ringens) by adding giant squid arm \\ surimi (Dosidicus gigas)
}

\author{
Santos Maza-Ramírez ${ }^{1}$, Daniel Pariona-Velarde ${ }^{1.2}$
}

\section{RESUMIEN}

El objetivo de este trabajo fue mejorar las propiedades funcionales del surimi de anchoveta mediante la adición de surimi de brazo de calamar gigante. Se evaluaron las características de textura y color de seis proporciones de surimi de brazo de calamar gigante / surimi de anchoveta $(0 / 100,20 / 80,40 / 60,50 / 50,70 / 30,100 / 0)$. Los resultados mostraron que las características de textura del gel y de color mejoraron considerablemente para la mezcla de surimi con 70/30, obteniéndose $522.2 \mathrm{~g}$ de dureza, $8.2 \mathrm{~mm}$ de elasticidad, $429.5 \mathrm{~g} * \mathrm{~cm}$ de fuerza de gel, y los valores de $\mathrm{L}^{*} \mathrm{y}$ a* fueron de $74.30 \mathrm{y}-0.85$, respectivamente. Este resultado indica que la mezcla de surimi con $70 / 30$ es la proporción indicada para mejorar las propiedades de la textura y el color del gel del surimi de anchoveta. La proporción óptima fue corroborada mediante programación lineal, donde se obtuvo una mejora del precio del surimi de anchoveta por la adición del surimi de calamar gigante.

Palabras clave: surimi, Engraulis ringens, Dosidicus gigas, dureza, elasticidad

\section{Abstract}

The aim of this study was to improve the functional properties of anchovy surimi by adding giant squid arm surimi. Texture and colour characteristics of six proportions of giant squid arm surimi / anchovy surimi (0/100, 20/80, 40/60, 50/50, 70/30, 100/0) were evaluated. The results showed that the texture characteristics of the gel and colour improved considerably for the mixture of surimi with 70/30, obtaining $522.2 \mathrm{~g}$ of hardness, $8.2 \mathrm{~mm}$ of elasticity, $429.5 \mathrm{~g} * \mathrm{~cm}$ of gel strength, and the values of $\mathrm{L}^{*}$ and $\mathrm{a} *$ were 74.30 and -0.85 , respectively. This result indicates that the $70 / 30$ mixture of surimi with $70 / 30$ is

\footnotetext{
${ }^{1}$ Instituto Tecnológico de la Producción (ITP)-DIDITT, Callao, Perú

2E-mail: jodanielbs@gmail.com
}

Recibido: 16 de febrero de 2019

Aceptado para publicación: 18 de enero de 2020

Publicado: 31 de marzo de 2020 
the best proportion to improve the texture and colour properties of the anchovy surimi gel. The optimal ratio was corroborated by linear programming, where an improvement in the price of anchovy surimi was obtained by the addition of giant squid surimi.

Key words: surimi, Engraulis ringens, Dosidicus gigas, hardness, elasticity

\section{INTRODUCCIÓN}

El proceso de producción de surimi convencional consiste en lavados sucesivos de la carne de pescado para el retiro de compuestos solubles en agua, enzimas, sangre, grasa y, principalmente, para concentrar las proteínas miofibrilares. Estas se gelifican por efecto del tratamiento térmico, produciendo una textura elástica en el producto elaborado (Lanier, 2000).

Recursos como la merluza (Merlucius productus), caballa (Rastre-lliger kanagurta) y la sardina (Sardinella gibbosa, Sardinella albella) se han empleado en la producción de surimi, los cuales presentan menor capacidad de formación de gel (Buamard et al., 2015, Chaijan et al., 2004). A nivel nacional, Maza y Solari (2006) elaboraron surimi de anchoveta (Engraulis ringens), el cual presentó los problemas señalados por Hultin y Kelleher (2000) y Park (2000), relacionados a la elaboración de surimi de pescados de carne roja; esto es, menor porcentaje de proteínas miofibrilares, alto contenido de grasa, color más oscuro por la presencia de hemoproteínas, y de fácil oxidación (Chaijan, 2006).

Para mejorar las propiedades de textura de surimi de anchoveta se adiciona mejoradores del gel de grado alimentario, como almidón, clara de huevo, proteína de suero, proteína de soya o plasma bovino(Hamann et al., 1990; Chen et al., 1993; Park, 1994) y mejoradores que no son de grado alimentario como EDTA (Ngo et al., 2010). Asimismo, el dióxido de titanio
$\left(\mathrm{TiO}_{2}\right)$ ha sido utilizado como blanqueador de color (Maza y Llave, 2006); sin embargo, estos mejoradores pueden afectar el sabor, color y la textura, así como incrementar el costo del producto (Park, 1994; Hsu et al., 1997; Hsu y Chiang, 2001). Una alternativa al uso de estos mejoradores en el surimi de anchoveta es mezclarlo con surimi obtenido a partir de manto, aleta y brazo de calamar gigante de color blanco (Dosidicus gigas), que tiene buena capacidad formadora de gel.

El objetivo de esta investigación fue mejorar y optimizar la textura y color del gel de surimi comercial de anchoveta mediante la adición de surimi de brazo de calamar gigante.

\section{Materiales y Métodos}

\section{Surimi Comercial de Anchoveta}

Se adquirieron muestras de $20 \mathrm{~kg}(10$ $\mathrm{kg} / \mathrm{bloque}$ ) de surimi de anchoveta de la Empresa «Arcopa» que se encontraban almacenados por dos meses en congelación. Los datos del proveedor fueron humedad: $77 \pm$ $1 \%$; fuerza de gel: 0-200 g/cm (Surimi tipo B); elasticidad: $>5 \mathrm{~mm}$; color: $\mathrm{L}: 67 \pm 1 ; \mathrm{b}<8$; impurezas: 10/g; pH: 6.6-7.5; lípido: 1.3\%; proteína mínima: $13 \%$; cenizas: $<0.6 \%$; histamina: $<100$ ppm; sodio: $919.2 \mathrm{mg} / \mathrm{kg}$. Así mismo, muestras de $20 \mathrm{~kg}$ (10 kg/bloque) de brazos congelados de calamar gigante fueron adquiridas de la misma empresa. Ambas muestras fueron almacenadas a $-25{ }^{\circ} \mathrm{C}$ hasta su uso. Los insumos de grado alimentario fueron cloruro sódico, cloruro de calcio, bicarbonato sódico. 


\section{Surimi de Brazo de Pota y Surimi Mez- clas}

Para la preparación de surimi de brazo de calamar gigante, el músculo descongelado, libre de piel y cortado en pequeñas piezas fue colocado en un mezclador-cortador de platillo (Yanagiya[J] SC-80H) con adición directa de ácido cítrico (Patente N. ${ }^{\circ}$ 7493, 07/ 05/2015). Los componentes solubles fueron removidos por prensado a $10 \mathrm{~kg} / \mathrm{cm}^{2}$. La proteína ácida concentrada fue neutralizada con la adición de bicarbonato de sodio ( $2 \%$ con respecto a la masa obtenida). Se aplicaron crioprotectores (4\% de sacarosa, $4 \%$ de sorbitol, $0.3 \%$ de mezcla de fosfatos $(50 / 50$ : pirofosfato/tripolifosfato de sodio) y 50 $\mathrm{mM}$ de $\mathrm{CaCl}_{2}$ en $91.7 \%$ de masa neutralizada) para su conservación en congelación $\left(-25^{\circ} \mathrm{C}\right)$

\section{Geles de Surimi}

Para la obtención de geles de surimi se preparó un coloide sol a partir de los surimis de brazo de pota (100:0), de anchoveta (0:100), y mezclas de estos, en proporciones de 20:80, 40:60, 50:50, 70:30 con surimi de brazo de calamar gigante y anchoveta, respectivamente. Obteniéndose así seis tipos de surimis. Cada uno de estos fue mezclado con $1.5 \%$ de $\mathrm{NaCl}$ durante 10 minutos en un mezclador-cortador de platillo. Se adicionó hielo para ajustar la humedad final al $72 \%$, manteniendo la temperatura menor a $10^{\circ} \mathrm{C}$.

La mezcla, libre de burbujas de aire, se dispuso en cilindros de acero inoxidable (3.0 $\mathrm{cm}$ diámetro y $3.5 \mathrm{~cm}$ de altura) y fue cocida a $90{ }^{\circ} \mathrm{C}$ en baño de agua durante 20 minutos, seguido de un enfriamiento en agua helada a $1{ }^{\circ} \mathrm{C}$ durante 10 minutos. Los geles de surimi se guardaron en bolsa ziplock a $5{ }^{\circ} \mathrm{C}$ por una noche.

\section{Prueba de Punción}

La prueba de punción se realizó en los geles con un analizador de textura Brookfield CT3-1500 (Brookfield Engineering Labora- torios, EEUU), comprimiendo geles de surimi (diámetro $=30 \mathrm{~mm}$, altura $=25 \mathrm{~mm}$ ) a una velocidad de $60 \mathrm{~mm} /$ minuto con una sonda esférica de $5 \mathrm{~mm}$ de diámetro hasta su ruptura. Las lecturas de los geles se realizaron después de 24 horas almacenados a $5{ }^{\circ} \mathrm{C}$. Se determinó la elasticidad $(\mathrm{cm})$, dureza $(\mathrm{g})$ y fuerza de gel $(\mathrm{cm} / \mathrm{g})$. Para la evaluación del grado de plegado, los geles se cortaron en rodajas de $3 \mathrm{~mm}$ de grosor, analizándose según lo recomendado por Nippon Suisan Kaisha (Chong, 1984).

\section{Prueba de Color}

El análisis de color se realizó en los geles mediante un colorímetro Nippon Denshoku ZE-200, utilizando la escala de valores del espacio de color CIELAB; L*denominada luminosidad (0 a 100); valor a*, proporción rojo (+) a verde (-) y el valor $b^{*}$, proporción amarillo $(+)$ a azul, además del cálculo de $\mathrm{L}^{*}$ $3 b^{*}$. Se determinó el índice de blancura con la ecuación: $\mathrm{IB}=100-\ll\left[\left(100-\mathrm{L}^{*}\right)^{2}+\mathrm{a}^{* 2}\right.$ $\left.\left.+\mathrm{b}^{* 2}\right]\right)$, y la diferencia total del color $(\Delta \mathrm{E}) \mathrm{de}$ cada surimi mezcla en relación al surimi de anchoveta se determinó con la ecuación $\Delta \mathrm{E}$ $=\mathrm{V}\left[\left(\mathrm{L}_{\mathrm{x}}^{*}-\mathrm{L}_{\mathrm{y}}{ }^{*}\right)^{2}+\left(\mathrm{a}_{\mathrm{x}}{ }^{*}-\mathrm{a}_{\mathrm{y}}{ }^{*}\right)+\left(\mathrm{b}_{\mathrm{x}}{ }^{*}-\mathrm{b}_{\mathrm{y}}{ }^{*}\right]\right)$, donde $x$ : valores del surimi de anchoveta y $y$ : valores del surimi mezcla.

\section{Análisis Estadístico}

Se aplicó el análisis de varianza y pruebas de comparaciones múltiples LSD a los valores de textura (fuerza de gel, dureza, elasticidad y grado de plegado) y a los valores $L^{*}, a^{*}, b^{*}$, IB. Así mismo, el análisis de regresión lineal para las características de textura o colorimétrica. Se utilizó el programa estadístico Statgraphics v. 5.0 para Windows. Todos los análisis fueron evaluados con un nivel de significancia de $\mathrm{p}<0.05$.

\section{Análisis de Optimización mediante Pro- gramación Lineal}

El proceso de optimización mediante programación lineal se desarrolló a base de las características iniciales de los surimis de 
brazo de calamar gigante, anchoveta y sus mezclas. A fin de maximizar el precio de venta del surimi de anchoveta, posibilitando la revalorización del surimi de anchoveta tipo $B$ mediante la mejora de las características de textura, plegado y de luminosidad. Las ecuaciones de restricción se desarrollaron según la fórmula de Yoon (1997): Propiedad del surimi mezcla $=(\% \mathrm{SA}) \mathrm{x}($ Propiedad SA $)$ $+(\%$ SBP $) \times($ Propiedad del SBP $)$, donde los valores de la restricción fueron: luminosidad $>70$, elasticidad $>8,450>$ fuerza de gel $>400$ y plegado $\geq 4$.5. Se utilizó el programa Classic LINDO $^{\mathrm{TM}}$ para evaluar la mezcla adecuada en función de los precios de venta.

\section{Resultados y Discusión}

\section{Composición Proximal}

La composición del brazo de calamar gigante presentó un contenido proteico de $17.4 \%$ y menor contenido de humedad de $80.4 \%$ (Cuadro 1), estando dentro del rango de proteína [11.5-17.45\%] y humedad [80.4 -85.3] presente en el manto de calamar (Hurtado, 2014). El contenido de proteína del brazo podría ser semejante al manto, pero la estructura del músculo es diferente debido a su función biológica para la captura de su presa y natación. La musculatura de estos apéndices es de estructura estriada oblicua y cruzada, formando filamentos gruesos y sarcómeros inusualmente cortos, lo cual se refleja en la elasticidad y velocidad de contracción (Kier 2016). Por otro lado, el surimi de anchoveta presentó un mayor contenido de humedad y grasa, y menor contenido de proteína que el surimi de brazo de calamar gigante (Cuadro 1) debido a su condición de especie pelágica.

\section{Textura de los Geles de Surimi Mezcla}

Los valores de dureza, elasticidad, fuerza de gel, plegado, valores de L* y b* del surimi de brazo de calamar gigante $(100 \%)$ presentaron diferencias significativas $(p<0.05)$ con aquellos del surimi de anchoveta $(100 \%)$ a consecuencia de la variación del porcentaje de proteína y bajo nivel de grasa; pero, además, debido a las posibles diferencias en cantidad de miosina, paramiosina y tejido conectivo solubilizado que se presentan durante la obtención del surimi, los cuales no están presentes en el surimi de anchoveta. La proporción de surimi 70:30 (brazo de calamar gigante: anchoveta) presentó los mejores valores reológicos, superando el valor de $400 \mathrm{~g} * \mathrm{~cm}$ de fuerza de gel (Cuadro 2).

El surimi de brazo de calamar gigante presentó buena capacidad de formación de gel, con elevada dureza, fuerza de gel y plegado de grado AA (Cuadro 2), siendo superior al surimi de manto de calamar gigante (348 g de dureza y $206 \mathrm{~g} * \mathrm{~cm}$ de fuerza de gel) reportado por Maza et al. (2003). Así mismo, se confirmó que el surimi de anchoveta presentó valores correspondientes a un surimi grado B (Arcopa).

\section{Color de los Geles de Surimi Mezcla}

El color del gel de surimi de anchoveta presentó mayor valor en la lectura de $\mathrm{b}^{*} \mathrm{y}$ menor valor $\mathrm{L}^{*}$ comparado con gel de surimi de brazo de calamar gigante. Choi y Park (2002) reportaron la disminución de blancura en el surimi por el matiz b* debido a la presencia de hemoglobina, mioglobina y compuestos de lípidos. Este mismo comportamiento se apreció en el surimi de anchoveta comercial del presente estudio, donde se mejoraron los valores de luminosidad con la mezcla de surimi de brazo con surimi de anchoveta (Cuadro 3).

Todos los parámetros de color de gel de surimi mezcla mostraron cambios significati$\operatorname{vos}(p<0.05)$. La mayor adición de surimi de brazo de calamar gigante mejora el color de la mezcla, excepto el valor de $b^{*}$ que no mostró cambio significativo, aunque indica una leve disminución en el aspecto de matiz amarillo. Este cambio del valor de b* en el gel de mezcla de surimi fue en la formulación óptima (70/30). En forma similar, aumentaron los valores de $\mathrm{L}^{*}$, índice de blancura y $\mathrm{L}^{*}-3 b^{*}$, 
Cuadro 1. Composición proximal de carne molida y surimi de brazo de calamar gigante, y de surimi de anchoveta

\begin{tabular}{lccc}
\hline \multirow{2}{*}{ Componentes (\%) } & \multicolumn{2}{c}{ Brazo de calamar gigante } & Anchoveta \\
\cline { 2 - 4 } & Músculo molido & Surimi & Surimi comercial* \\
\hline Proteína & $17.4 \pm 0.5$ & $14.1 \pm 0.3$ & 13.0 \\
Humedad & $80.4 \pm 0.2$ & $75.4 \pm 0.5$ & 77.0 \\
Grasa & $0.5 \pm 0.3$ & $0.4 \pm 0.1$ & 1.3 \\
Ceniza & $2.3 \pm 0.04$ & $2.3 \pm 0.02$ & 0.5 \\
\hline
\end{tabular}

* Surimi comercial de grado B, reportado por Arcopa

Cuadro 2. Parámetros de textura de gel surimi mezcla (brazo de calamar gigante/anchoveta)

\begin{tabular}{ccccc}
\hline $\begin{array}{c}\text { Brazo de calamar } \\
\text { gigante } / \\
\text { anchoveta }\end{array}$ & Dureza $(\mathrm{g})$ & $\begin{array}{c}\text { Elasticidad } \\
(\mathrm{mm})\end{array}$ & $\begin{array}{c}\text { Fuerza de gel } \\
\left(\mathrm{g}^{*} \mathrm{~cm}\right)\end{array}$ & $\begin{array}{c}\text { Grado de plegadc } \\
(\text { puntaje japonés) }\end{array}$ \\
\cline { 2 - 5 } $0 / 100$ & $251.0 \pm 25.9^{\mathrm{a}}$ & $6.6 \pm 0.4^{\mathrm{a}}$ & $166.0 \pm 21.6^{\mathrm{a}}$ & A (4.0) \\
$20 / 80$ & $278.0 \pm 31.5^{\mathrm{a}}$ & $6.7 \pm 0.4^{\mathrm{a}}$ & $187.0 \pm 28.7^{\mathrm{a}, \mathrm{b}}$ & $\mathrm{A}(4.0)$ \\
$40 / 60$ & $309.0 \pm 41.1^{\mathrm{b}}$ & $7.0 \pm 0.4^{\mathrm{b}}$ & $217.0 \pm 36.4^{\mathrm{b}}$ & $\mathrm{A}(4.0)$ \\
$50 / 50$ & $387.0 \pm 47.0^{\mathrm{c}}$ & $7.3 \pm 0.4^{\mathrm{c}}$ & $286.0 \pm 49.0^{\mathrm{c}}$ & A (4.4) \\
$70 / 30$ & $522.2 \pm 49.7^{\mathrm{d}}$ & $8.2 \pm 0.6^{\mathrm{d}}$ & $429.5 \pm 69.9^{\mathrm{d}}$ & $\mathrm{AA}(4.7)$ \\
$100 / 0$ & $646.6 \pm 73.5^{\mathrm{d}}$ & $8.9 \pm 0.7^{\mathrm{e}}$ & $574.6 \pm 89.8^{\mathrm{e}}$ & AA (5.0) \\
\hline
\end{tabular}

$a, b$ Letras diferentes dentro de columnas indican diferencias significativas $(p<0.05)$

Cuadro 3. Parámetros de color de los geles de surimi mezcla (surimi de brazo de calamar gigante y surimi de anchoveta)

\begin{tabular}{ccccccc}
\hline \multirow{2}{*}{$\begin{array}{c}\text { Surimi } \\
\text { brazo de calamar } \\
\text { gigante / anchoveta }\end{array}$} & $\mathrm{L}^{*}$ & $\mathrm{a}^{*}$ & $\mathrm{~b}^{*}$ & $\begin{array}{c}\text { Índice de } \\
\text { blancura }\end{array}$ & $\mathrm{L}^{*}-3 \mathrm{~b}^{*}$ & $\Delta \mathrm{E}$ \\
\cline { 2 - 7 } $0 / 100$ & $62.85 \pm 0.10^{\mathrm{a}}$ & $-0.15 \pm 0.12^{\mathrm{a}}$ & $15.36 \pm 0.05^{\mathrm{a}, \mathrm{b}}$ & $59.80 \pm 0.08^{\mathrm{a}}$ & $16.77 \pm 0.08^{\mathrm{a}}$ & 0 \\
$20 / 80$ & $66.46 \pm 0.22^{\mathrm{b}}$ & $-0.61 \pm 0.09^{\mathrm{b}, \mathrm{c}}$ & $15.61 \pm 0.88^{\mathrm{a}}$ & $15.61 \pm 0.88^{\mathrm{a}}$ & $19.64 \pm 2.46^{\mathrm{b}}$ & $3.72 \pm 0.20^{\mathrm{a}}$ \\
$40 / 60$ & $68.86 \pm 0.36^{\mathrm{c}}$ & $-0.53 \pm 0.15^{\mathrm{c}}$ & $15.99 \pm 0.33^{\mathrm{a}}$ & $15.99 \pm 0.33^{\mathrm{a}}$ & $20.89 \pm 1.35^{\mathrm{b}}$ & $6.06 \pm 0.34^{\mathrm{b}}$ \\
$50 / 50$ & $70.33 \pm 0.37^{\mathrm{d}}$ & $-0.76 \pm 0.04^{\mathrm{b}, \mathrm{d}}$ & $15.65 \pm 0.30^{\mathrm{a}}$ & $15.65 \pm 0.30^{\mathrm{a}}$ & $23.39 \pm 1.24^{\mathrm{c}}$ & $7.51 \pm 0.35^{\mathrm{c}}$ \\
$70 / 30$ & $74.30 \pm 0.54^{\mathrm{e}}$ & $-0.85 \pm 0.10^{\mathrm{d}, \mathrm{e}}$ & $14.83 \pm 0.32^{\mathrm{d}}$ & $14.83 \pm 0.32^{\mathrm{d}}$ & $29.81 \pm 0.53^{\mathrm{d}}$ & $11.48 \pm 0.52^{\mathrm{d}}$ \\
$100 / 0$ & $80.37 \pm 0.33^{\mathrm{f}}$ & $-0.99 \pm 0.14^{\mathrm{e}}$ & $11.41 \pm 0.15^{\mathrm{e}}$ & $11.41 \pm 0.15^{\mathrm{e}}$ & $46.15 \pm 0.64^{\mathrm{e}}$ & $17.98 \pm 0.34^{\mathrm{e}}$ \\
\hline
\end{tabular}

$\Delta \mathrm{E}$ : Diferencia total de color

$a, b, c, d, e$ Letras diferentes dentro de columnas indican diferencias significativas $(p<0.05)$ 
Cuadro 4. Consideraciones para uso del programa de optimización de la mezcla de surimi de brazo de calamar gigante y de anchoveta

\begin{tabular}{ll}
\hline Función objetivo & MAX: $2.5 * \mathrm{SBP}+1.497 * \mathrm{SA}$ \\
\hline Restricciones de proporción: & $1>\mathrm{SBP} \geq 0.1$ \\
& $1>\mathrm{SA} \geq 0.1$ \\
& $\mathrm{SBP}+\mathrm{SA}=1$ \\
Restricciones de luminosidad: & $62.85 * \mathrm{SA}+80.37 * \mathrm{SBP}>70$ \\
Restricciones de elasticidad: & $6.62 * \mathrm{SA}+8.90 * \mathrm{SBP}>8$ \\
Restricciones de fuerza de gel: & $450>166 * \mathrm{SA}+574.6 * \mathrm{SBP}>400$ \\
Restricciones de plegado: & $4 * \mathrm{SA}+5 * \mathrm{SBP} \geq 4.5$ \\
\hline
\end{tabular}

SBP: Proporción de peso de surimi de brazo de calamar gigante

SA: proporción de peso de surimi de anchoveta

El precio de SBP y SA fue US\$2.5 (precio estimado) y US\$1.497 por kg, respectivamente

siendo más próximos a valor de $100 \%$ de surimi de brazo de calamar gigante. La diferencia total del color $(\Delta \mathrm{E})$ con respecto al surimi de anchoveta $(100 \%)$ se incrementó a medida que la proporción de surimi de calamar gigante aumentaba, mejorando significativamente los valores de luminosidad e índice de blancura.

\section{Optimización de Mezcla de Surimi (Pro- grama Lindo)}

La optimización lineal fue utilizada para la maximización de precio de la mezcla de surimi en referencia a la textura (dureza, elasticidad, plegado) y luminosidad ( $\left.\mathrm{L}^{*}\right)$ de su gel evaluado como se muestra en la Cuadro 4. El programa indica que la mezcla óptima fue $69.5 \%$ de surimi de brazo de calamar gigante con $30.5 \%$ de surimi de anchoveta. Estos resultados concuerdan con los datos experimentales mostrados en la Cuadro 3 (proporción 70:30). El precio estimado de la mezcla óptima fue $\$ 2.19 / \mathrm{kg}$. Esta proporción se mantendrá siempre y cuando solo uno de los precios varíe en $\pm \$ 1.00$.

\section{Conclusiones}

- La textura y color del surimi de anchoveta tipo B fue mejorado utilizando surimi de brazo de calamar gigante.

- La proporción óptima fue de 70:30 (brazo de calamar gigante: anchoveta). Las características de textura fueron: elasticidad de $8.2 \mathrm{~mm}$; dureza $522 \mathrm{~g}$ y fuerza de gel 429 g.cm, plegado AA, con valores de $L^{*}=74.3 ; a^{*}=-0.85 ; b^{*}=14.83$.

- Los resultados experimentales de textura y color coincidieron con los resultados obtenidos con el programa de optimización aplicado, donde en ambos casos se indicó como mezcla óptima la de 70:30 (brazo de calamar gigante: anchoveta).

\section{Agradecimientos}

Al Instituto Tecnológico de la Producción (ITP) por las facilidades al desarrollar este trabajo de investigación. 


\section{Literatura Citada}

1. Buamard N, Benjakul S. 2015. Improvement of gel properties of sardine (Sardinella albella) surimi using coconut husk extracts. Food Hydrocolloids 51: 146-155. doi:10.1016/ j.foodhyd.2015.05.011

2. Chaijan M. 2006. Characterization of muscle proteins and interaction between myofibrillar proteins and myglobin of Dark-Fleshed fish used for surimi production. Doctoral Thesis. Thailand: Prince of Songkla University. 202 p.

3. Chaijan M, Benjakul S., Visessanguan W, Faustman C. 2004. Characteristics and gel properties of muscles from sardine (Sardinella gibbosa), and mackerel (Rastrelliger kanagurta) caught in Thailand. Food Res Int 37:1021-1030. doi: 10.1016/j.foodres.2004.06.012

4. Chen J, Lee C, Crapo C. 1993. Linear programming and response surface methodology to optimize surimi gel texture. J Food Sci 58: 535-538. doi: 10.1111/j.1365-2621.1993.tb04318.x

5. Chong L. 1984. Surimi process technology. Food Technol 38: 69-80.

6. Choi Y, Park J. 2002. Acid-aided protein recovery from enzyme-rich Pacific whiting. J Food Sci 67: 29622967. doi: 10.1111/j.1365-2621.2002.tb08846.x

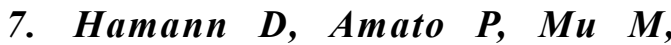
Foegeding E. 1990. Inhibition of modori (gel weakening) in surimi by plasma hydrolysate and egg white. J Food Sci 55: 665-669. doi: 10.1111/j.13652621.1990.tb05202.x

8. Hultin H, Kelleher S. 2000. Surimi proce-ssing from dark muscle ûsh. In: Park JW (ed). Surimi and surimi seafood. New York, USA: Marcel Dekker. p 59-77.

9. Hurtado L. 2014. Elaboración de pasta untable a partir de recortes de pota (Dosidicus gigas) en envase de $1 / 4$ club. Tesis de Ingeniero Pesquero. Lima: Univ. Nacional Agraria La Molina. 130 p.
10. Hsu C, Kolbe E, English M. 1997. A nonlinear programming technique to develop least cost formulations of surimi products. Food Process Eng 20: 179-196. doi: 10.1111/j.1745-4530.1997.tb00418.x

11. Hsu C, Chiang B. 2001. Optimum blends of different grades of surimi determined by non-linear programming. Fisheries Sc 67: 500-505. doi: 10.1046/ j.1444-2906.2001.00264.x

12. Kier $W$. 2016. The musculature of coleoid cephalopod arms and tentacles. Front Cell Dev Biol 4:10. doi: 10.3389/ fcell.2016.00010

13. Lanier TC. 2000. Surimi gelation chemistry. In: Park JW (ed). Surimi and surimi seafood. New York, USA: Marcel Dekker. p 237-265.

14. Maza S, Rosales M, Castro R. 2003. Efecto de un proceso de lixiviación ácida salina sobre la calidad del surimi de Dosidicus gigas «Calamar gigante». Bol Invest Inst Tecnol Pesq Perú 5: 81-88. [Internet]. Disponible en: http:// repositorio.itp.gob.pe/handle/ITP/103

15. Maza S, Llave I. 2005. Mejoramiento del color de pulpa y surimi de anchoveta peruana (Engraulis ringens). Bol Invest Inst Tecnol Pesq Peru. 7: 1-10. [Internet]. Disponible en: http:// repositorio.itp.gob.pe/handle/ITP/69

16. Maza S, Solari A. 2006. Influencia del tiempo, temperatura y refinación en la textura del surimi de anchoveta peruana (Engraulis ringens). Bol Invest Inst Tecnol Pesq Perú 7: 11-19. [Internet]. Disponible en: http://reposito-rio.itp.gob.pe/handle/ITP/70

17. Maza S. 2015. Procedimiento de obtención de un concentrado proteico deodorizado por método de triturado de los recortes de manto de calamar gigante (Dosidicus gigas) con ácido cítrico, sin uso de agua. Patente Otorgada N. ${ }^{\circ} 7493$, 07/05/2015. [Internet]. Disponible en: https://servicio.indecopi.gob.pe/portalSAE/Expedientes/consulta-OIN.jsp?pListar $=\& p N r o E-x p e-d i e n t e=651 \& p-$ A n i o Expe - d i e n t e $=2014$ $\&$ Captcha $=u u 2 z$ 
18. Ngo V, Katsuji M, Yoshiaki I. 2010. Gel-forming characteristics of surimi from white croaker under the inhibition of the polymerization and degradation of protein. J Biol Sci 10: 432-439. doi: 10.3923/jbs.2010.432.439

19. Park J. 1992. Use of various grades of surimi with an application of least cost formulation. In: Sylvia GMorrissey MT (eds). Paciûc whiting: harvesting, proces-sing, marketing, and quality assurance. Oregon, USA. Oregon State University. p 17-19.

20. Park J. 1994. Functional protein additives in surimi gels. J Food Sci 59: 525-527. doi: 10.1111/j.1365-2621.1994.tb05554.x
21. Park J. 2000. Surimi seafood: products, market, and manufacturing. In: Park JW (ed). Surimi and surimi seafood. New York, USA: Marcel Dekker. p 201-235.

22. Lindo Systems. 2019. LINDO Software for integer programming, linear programming, nonlinear programming, stochastic programming, global optimization. [Internet]. Available in: www.lindo.com

23. Yoon Y, Kim B, Park J. 1997. Linear programming in blending various components of surimi seafood. J Food Sci 62: 564-567. doi: 10.1111/j.13652621.1997.tb04430.x 